\title{
MEROMORPHIC STABILIZATION AND CONTROL OF TIME DELAY SYSTEMS
}

\author{
Pavel Zítek, Vladimír Kučera and Tomáš Vyhlídal
}

\author{
Center for Applied Cybernetics, \\ Faculty of Mechanical Engineering, Faculty of Electrical Engineering, \\ Czech Technical University in Prague, Technická. 4, 16607 Praha 6, Czech Republic \\ zitek@fsid.cvut.cz,kucera@fel.cvut.cz,vyhlidal@fsid.cvut.cz
}

\begin{abstract}
The objective of the paper is to overcome the conventional restriction to the field of rational functions in the algebraic design of control systems. By combining a shift of the undesirable poles to the left with an extension of an inverse-based affine parameterization approach, an algebraic solution to time-delay system stabilization and control is opened for plants and controllers modeled by the so-called RQ-meromorphic functions, thus relaxing the often used assumption of commensurate delays. The design proposed results in an internally stable control loop involving both integrators and delayers in the controller structure. Owing to the use of time-shifted data, this kind of controller is referred to as anisochronic. Copyright $\subseteq 2005$ IFAC
\end{abstract}

Keywords: inverse-based parameterization, retarded time-delay system, pole shifting, meromorphic function, stabilizing controller, Bézout equation

\section{INTRODUCTION}

The affine parameterization of stabilizing controllers has become a primary approach to the design of linear control systems described by rational transfer functions. However, constraining the algebraic control system design to the class of rational models turned out to be an undue restriction. The methods like finite spectrum assignment (Malek-Zavarei and Jamshidi, 1987; Wan, Lee and Tan, 1995), functional pole placement (Zítek et al., 2001) or functional extension of internal model control principle (Zítek and Hlava, 2001, Zítek and Vyhlidal, 2003), to name a few, have shown how a purposeful combination of integrators and delayors in controller structure can improve the feedback system properties.

The algebraic description of time delay systems requires a simultaneous use of both differential and delay operators. Using the transcendental functions for the algebraic design of time delay systems is quite natural in view of the fact that the rational functions are a subset of the class of meromorphic functions, well known in the complex analysis. The
Laplace-transform description of linear time-delay systems results in transfer functions that are ratios of the so-called quasi-polynomials, i.e. weighted sums of products of $s$-powers and exponential functions of $s$-multiples. In principle, the algebraic approaches to controller design, e.g. the pole placement approach or affine controller parameterization, may be adopted for this kind of infinite order systems, however, with serious constraints. Most of the problems following these extensions towards meromorphic functions are more or less connected with the transcendental nature of the models, i.e. with the infinite spectrum of these models. Stabilization and synthesis of the so-called fractional exponential systems has been worked out by Bonnet and Partington (1999). As regards the pole placement, it is severely limited by the fact that infinite number of poles is influenced by a low number of control parameters. Some of the achieved results have been presented by Michiels et al. (2002) or Michiels and Vyhlídal, 2004). Basically, the pole placement issue in the time delay systems should be interpreted rather as a kind of shifting the dominant (or the rightmost and undesirable) poles to the left (Michiels and Roose, 2001, Zítek and Vyhlídal, 
2002), since the majority of the infinite rest of the spectrum is placed spontaneously. The result of the pole shifting is significantly limited by the wellknown effect that the shifting of the rightmost poles to the left is mostly accompanied with a tendency to shift the rest of the spectrum to the right. In addition, the parameterization based controller design is subject to significant limitations due to much larger variety of quasi-polynomials and due to the causality requirements in the time delay systems (Zítek and Kučera, 2003).

\section{MEROMORPHIC EXTENSION OF CONTROLLER PARAMETERIZATION}

In extending the class of admissible functions from rational to meromorphic, the natural requirements of causality and feasibility of both the plant and the controller have to be respected in the ultimate control system implementation. To satisfy these conditions in rational algebraic design, one constrains the plant and controller models to proper rational functions. An equivalent restriction is to be introduced for meromorphic functions as well. In order to avoid impulsive modes in system's responses the so-called internal stability condition is adopted. To apply the algebraic approach to the feedback design of timedelay systems, it is necessary to define an admissible class of these systems, particularly as to the delays. The time-delay systems are supposed containing lumped delays only and with the so-called retarded structure (Hale and Verduyn Lunel, 1993). This class of systems is defined below.

Definition 1 (Retarded quasi-polynomial) A characteristic quasi-polynomial of a linear lumped-delay system is referred to as retarded one if it is of the generic form

$$
A(s)=s^{n}+\sum_{i=0}^{n-1} \sum_{j=1}^{h} a_{i j} s^{i} \exp \left(-\vartheta_{i j} s\right)
$$

i.e. its highest $s$-power $s^{n}$ represents a delayless term of the model and $\vartheta_{i j}$ are non-negative delays.

Definition 2 ( $R Q$ meromorphic function) A ratio of quasi-polynomials $B(s) / A(s)$ is said to be a retarded quasi-polynomial (RQ) meromorphic function if

- $A(s)$ is a retarded quasi-polynomial as in (1),

- $B(s)$ can be factorized as $B(s)=$ $=\widetilde{B}(s) \exp (-s \tau)$, where $\tau>0$ and $\widetilde{B}(s)$ is a retarded quasi-polynomial

$$
\widetilde{B}(s)=s^{m}+\sum_{i=0}^{m-1} \sum_{j=1}^{h} b_{i j} s^{i} \exp \left(-\tau_{i j} s\right),
$$

- the fraction is proper, i.e., it holds for the highest $s$-power $s^{m}$ in $B(s)$, that $m \leq n$.

Remark. The last requirement of properness is not directly connected with the notion of retarded quasi- polynomial fraction, but it declares the natural aim to work with the feasible models only.

Definition 3 (Asymptotic stability) An RQmeromorphic function is said to be asymptotically stable if it is analytic in the closed right half $s$-plane, i.e. if its denominator retarded quasi-polynomial $A(s)$ has no finite zero $s_{0}$ such that $\operatorname{Re} s_{0} \geq 0$.

Due to the infinite spectrum, of the $A(s)$ zeros, particularly the issue of pole placement is of a completely different nature. Only the stability of retarded quasi-polynomials can still be checked by the Mikhaylov criterion. In order to keep the utmost analogy between the conventional algebraic approach and the time-delay system design, the ring of RQmeromorphic stable functions, denoted by $\mathrm{R}_{M S}$, is introduced in the sequel.

The design of controllers based on the affine parameterization of stabilizing controllers can be well performed in the case of a stable plant even when the plant is described by an RQ meromorphic transfer function (Zítek and Kučera, 2003). In the case of an unstable plant with delays, however, the algebraic approach requires a good deal of inventiveness to succeed in finding the admissible quasi-polynomials. For this reason we propose to design the control system as a cascade connection of two controllers $R_{0}(s)$ and $R_{M}(s)$, see Fig. 1 .

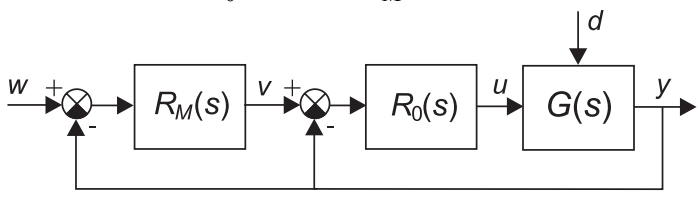

Fig. 1 Proposed cascade controller scheme

Hence the controller is designed in two steps:

- First a preliminary controller $R_{0}(s)$ is added to the plant, shifting the rightmost poles to the left to obtain a stable RQ meromorphic function

$$
H(s)=\frac{R_{0}(s) G(s)}{1+R_{0}(s) G(s)}
$$

to be controlled. If the preliminary feedback loop with $R_{0}(s)$ results in a stable $H(s)$ this transfer function can be considered as a modified or precompensated system to be controlled by the controller $R_{M}(s)$.

- Secondly, a master controller $R_{M}(s)$ is designed as a stable RQ meromorphic function, using the method of affine parameterization. This controller is designed to achieve a desirable performance of reference tracking and disturbance rejection, while advantage is taken of a simple affine parameterization for stable systems in this design phase.

The reason for combining the two methods is to avoid the weak points of each. On the one hand, the method of affine parameterization provides a wellfitted compensation for delays and other undesirable 
dynamics, but only if the system to be controlled is stable. For unstable time-delay systems, this approach is far from being feasible and the result depends critically on the inventiveness of the designer. On the other hand, the pole shifting has proved an effective tool to rid the system of the right half-plane poles. However, due to the infinite rest of the system spectrum, the final distribution of the entire spectrum cannot be guaranteed by this procedure and the possibility to compensate delays is rather limited.

\section{SHIFTING THE POLES OF THE PLANT}

Consider an unstable time-delay plant described by an RQ meromorphic transfer function $G(s)$ as in Definition 2. In addition, we assume that $m \leq n-1$. Let a preliminary stabilizing controller be designed with a proper rational transfer function

$$
R_{0}(s)=\frac{P(s)}{Q(s)}=\frac{p_{v} s^{v}+\ldots+p_{2} s^{2}+p_{1} s+p_{0}}{s^{v}+\ldots+q_{2} s^{2}+q_{1} s+q_{0}}
$$

where $v=n-1$. The characteristic quasi-polynomial of the preliminary control loop of the order $n+v=$ $=2 n-1$ is

$$
\begin{aligned}
& A(s) Q(s)+B(s) P(s)=M(s)=s^{2 n-1}+ \\
& +\sum_{i=0}^{2 n-2}\left(\sum_{j=0}^{n-2} q_{j} \gamma_{i j}(s)+\sum_{j=0}^{n-1} p_{j} \beta_{i j}(s)+\alpha_{i}(s)\right) s^{i}
\end{aligned}
$$

where the set of $2 n-1$ coefficients of $R_{0}(s)$ is considered as a parameter vector, $\mathbf{p}=\left[p_{0}, p_{1}, \ldots p_{v}, q_{0}, q_{1}, \ldots q_{v-1}\right]^{T}$ and $\alpha_{i}(s), \beta_{i j}(s)$, $\gamma_{i j}(s)$ are the functions resulting from the products $A(s) Q(s)$ and $B(s) P(s)$. Apparently, $M(s)$ is a retarded quasi-polynomial according to Definition 1. Therefore only a finite part of the zero spectrum of $M(s)$ is located at the rightmost position (Michiels et al., 2002) while the infinite rest of the spectrum, $r_{k}=\beta_{k} \pm j \omega_{k}$, tend to the left, satisfying $\lim \beta_{k}=-\infty$ as $k \rightarrow \infty$. Moreover, since $R_{0}(s)$ is a stabilizing controller, $M(s)$ is a stable retarded quasipolynomial, and $H(s)$ is a stable RQ meromorphic function. As shown in (Zítek and Kučera, 2003) the preliminary control loop with a controller (4) and with a stable characteristic quasi-polynomial (5) is not only stable but also even internally stable, meaning that all four closed-loop sensitivity functions are stable.

Proposition 1. Consider a preliminary control loop of $G(s)$ and $R_{0}(s)$ as in (4). By way of trial fix preliminarily a set of the parameters $\overline{\mathbf{p}}=$ $=\left[\bar{p}_{0}, \bar{p}_{1}, \ldots \bar{p}_{v}, \bar{q}_{0}, \bar{q}_{1}, \ldots \bar{q}_{v-1}\right]^{T}, \quad$ resulting in a characteristic quasi-polynomial $M=M(s, \overline{\mathbf{p}})$. Using a software tool as in Vyhlídal and Zítek, (2003), assess the rightmost part of the spectrum of, say, $N$ zeros of $M(s, \overline{\mathbf{p}})$, to be denoted as $\bar{r}_{k}, k=1,2, \ldots N$, where $N>2 n-1$. In order to shift a subset of $2 n-1$ rightmost zeros $\bar{r}_{k}$ to some more desirable new positions $r_{k}^{*}, k=1,2, \ldots 2 n-1$ the parameters $\overline{\mathbf{p}}$ are to be altered to new ones, denoted $\mathbf{p}^{*}$. The required change of parameters from $\overline{\mathbf{p}}$ to $\mathbf{p}^{*}$ can be achieved by solving the following set of equations

$$
\begin{aligned}
M\left(r_{k}^{*}, \overline{\mathbf{p}}\right)+\sum_{i=0}^{n-1} & \left.\frac{\partial M(s, \mathbf{p})}{\partial p_{i}}\right|_{s=r_{k}^{*}}\left(p_{i}^{*}-\bar{p}_{i}\right)+ \\
& +\left.\sum_{i=0}^{n-2} \frac{\partial M(s, \mathbf{p})}{\partial q_{i}}\right|_{s=r_{k}^{*}}\left(q_{i}^{*}-\bar{q}_{i}\right)=0
\end{aligned}
$$

for $k=1,2, \ldots 2 n-1$.

Proof. Consider the characteristic quasi-polynomial as a complex function of both the complex variable $s=\beta+j \omega$ and the vector of real parameters $\mathbf{p}$, $\mathbf{p} \in \mathrm{R}^{2 n-1}, M=M(s, \mathbf{p})$. Since $M$ is of the form (5), it is linear with respect to the parameters $\mathbf{p}$ and therefore the partial derivatives $\partial M / \partial p_{i}$ or $\partial M / \partial q_{i}, i=1,2, \ldots$ exist and depend only on $s$, not on the parameter vector. For a trial setting of parameters $\mathbf{p}=\overline{\mathbf{p}}$ the appropriate quasi-polynomial form is $M=M(s, \overline{\mathbf{p}})$ and its spectrum of zeros is $\bar{r}_{k}, k=1,2, \ldots \infty$. By linearity, the change of $M(s, \mathbf{p})$ brought about by a change $\Delta \mathbf{p}$ of the parameters, is given by the differential form

$$
\Delta M(s, \mathbf{p})=\left.\frac{\partial M(s, \overline{\mathbf{p}})}{\partial \mathbf{p}}\right|_{s=s^{*}} \Delta \mathbf{p}
$$

where $s=s^{*}$ is the point where the change is to be assessed and

$$
\frac{\partial M(s, \mathbf{p})}{\partial \mathbf{p}}=\left[\begin{array}{lll}
\frac{\partial M(s, \mathbf{p})}{\partial p_{1}}, & \ldots & \frac{\partial M(s, \mathbf{p})}{\partial q_{v-1}}
\end{array}\right]
$$

is the gradient of $M$ in $\mathrm{R}^{2 n-1}$. Consider still the quasi-polynomial form $M=M(s, \overline{\mathbf{p}})$ and try to find a new parameter setting $\mathbf{p}^{*}$ for which the new position of the $M$ zeros is $r_{k}^{*}$ instead of $\bar{r}_{k}$. Suppose the number of placed zeros $r_{k}^{*}$ is equal to the number of parameters, $k=1,2, \ldots 2 n-1$. Inserting $s=r_{k}^{*}$ into (7) and realizing that $M\left(r_{k}{ }^{*}, \mathbf{p}^{*}\right)=0$ the following condition is obtained

$$
0=M\left(r_{k}^{*}, \overline{\mathbf{p}}\right)+\left.\frac{\partial M(s, \mathbf{p})}{\partial \mathbf{p}}\right|_{s=r_{k}^{*}}\left(\mathbf{p}^{*}-\overline{\mathbf{p}}\right),
$$


$k=1,2, \ldots, 2 n=1$, where $\mathbf{p}^{*}$ is the new vector of parameters to be assessed. As soon as (9) is satisfied for each of the prescribed roots $s=r_{k}^{*}$, $k=1, \ldots, 2 n-1$, the set (6) is obtained. Thanks to linearity, this equation gives the true $M$ increment without any constraint on the size of the parameter change.

Example 1. An unstable time delay plant described by the second-order model

$$
G(s)=\frac{\exp (-s \tau)}{s^{2}-a \exp (-s \vartheta)},
$$

is given and a stabilizing controller is to be designed. According to (4), consider a preliminary controller of the form $R_{0}(s)=(\delta s+\kappa) /(s+\lambda)$, where $\lambda, \delta, \kappa$ are the parameters. With the plant data $a=0.5, \tau=0.5$, $\vartheta=0.2$, the characteristic quasi-polynomial of the auxiliary control loop is

$$
\begin{aligned}
& M(s, \mathbf{p})=s^{3}+\lambda s^{2}+(\delta \exp (-0.5 s)- \\
& -0.5 \exp (-0.2 s)) s+\kappa \exp (-0.5 s)- \\
& -0.5 \lambda \exp (-0.2 s)
\end{aligned}
$$

where $\mathbf{p}=[\lambda, \delta, \kappa]^{T}$. With the trial setting of parameters $\lambda=2, \delta=2, \kappa=2$, the first part of the $M(s, \overline{\mathbf{p}})$ zeros is as follows: $\bar{r}_{1}=-1.1705$, $\bar{r}_{2,3}=-0.0417 \pm j 0.8284, \quad \bar{r}_{4,5}=-8.782 \pm j 9.758$, $\bar{r}_{6,7}=-11.640 \pm j 23.345, \bar{r}_{8,9}=-13.219 \pm j 36.364$, $\bar{r}_{4,5}=-14.344 \pm j 49.163$, etc. The rightmost zeros $\bar{r}_{1,2,3}$ are to be shifted to the new positions: $r_{1}^{*}=-0.5, r_{2}^{*}=-1, r_{3}^{*}=-1.5$. To form equation set (6) the following derivatives

$$
\begin{gathered}
\frac{\partial M(s, \mathbf{p})}{\partial \lambda}=s^{2}-0.5 \exp (-0.2 s) \\
\frac{\partial M(s, \mathbf{p})}{\partial \delta}=s \exp (-0.5 s), \frac{\partial M(s, \mathbf{p})}{\partial \delta}=\exp (-0.5 s)
\end{gathered}
$$

as well as $M(s, \overline{\mathbf{p}})$ have been evaluated for the values $s=r_{1}^{*} \quad s=r_{2}^{*}$ and $s=r_{3}^{*}$. The following equations are obtained for $s=\bar{r}_{1}{ }^{*}$

$$
\begin{aligned}
& 0.8301-0.3026 \Delta \lambda-0.6420 \Delta \delta+ \\
& +1.2840 \Delta \kappa=0
\end{aligned}
$$

and for $s=\bar{r}_{2}^{*}$

$$
\begin{aligned}
& -0.2109-j 0.2925+(-0.6214-j 0.0343) \Delta \lambda+ \\
& +(-0.0395+j 0.3806) \Delta \delta+ \\
& +(1.0352-j 0.1830) \Delta \kappa=0+j 0
\end{aligned}
$$

The last condition gives two equations for the real and the imaginary parts

$$
-0.2109-0.6214 \Delta \lambda-0.0395 \Delta \delta+1.0352 \Delta \kappa=0
$$

$$
-0.2925-0.0343 \Delta \lambda+0.3806 \Delta \delta-0.1830 \Delta \kappa=0
$$

From (12), (15), (16) the following parameters are obtained: $\quad \lambda=2+\Delta \lambda=14.5636, \quad \delta=2+\Delta \delta=$ $=13.0336, \kappa=2+\Delta \kappa=9.8309$. The first three roots are located as specified, however, the complete spectrum of $r_{k}^{*}$ is different from the initial one as well: $\quad r_{4,5}^{*}=-5.6716 \pm j 12.8414, \quad r_{6,7}^{*}=-7.9234$ $\pm j 24.9783, r_{8,9}^{*}=-9.4451 \pm j 374430$, etc. It is worth noting that the entire root chain is shifted to the right, i.e., towards the stability boundary.

\section{AFFINE PARAMETERIZATION BASED CONTROLLER DESIGN}

Having selected a fitting set of parameters $\mathbf{p}$ for the pre-compensating rational controller $R_{0}(s)$, a stable time-delay system is obtained described by RQ meromorphic transfer function

$$
H(s)=\frac{R_{0}(s) G(s)}{1+R_{0}(s) G(s)}
$$

considered as a modified plant to be controlled, where the orders of the denominator and the numerator are $2 n-1$ and $m+n-1$, respectively. The stability of $H(s)$ renders the affine parameterization approach applicable in designing the master controller $R_{M}(s)$. Consider the master controller as a meromorphic function to have more degrees of freedom in compensating the transcendental terms of $H(s)$. The affine parameterization is based on the general idea of plant inversion, assuming the control feedback scheme shown in Fig. 2. The controller performance is adjusted through a parameterizing control function $C_{R}(s)$ (Goodwin et al., 2001) with the aim of obtaining the reference-to-output transfer function in the product form

$$
T(s)=\frac{y(s)}{w(s)}=C_{R}(s) H(s)
$$

A perfect tracking transfer function, $T(s)=1$, could be achieved only in the ideal case, where $C_{R}(s)$ would invert completely the real plant model. Using the parameterizing controller function $C_{R}(s)$, the control scheme in Fig. 2 can be transformed to a single feedback controller with an equivalent master controller transfer function

$$
R_{M}(s)=\frac{C_{R}(s)}{1-C_{R}(s) H(s)}
$$

It already has been proved that for $H(s) \in \mathrm{R}_{M S}$ the control system structured as in Fig. 2 is internally stable if and only if the parameterizing control 
function $C_{R}(s)$ is any stable function in $\mathrm{R}_{M S}$ (Zítek and Kučera, 2003).

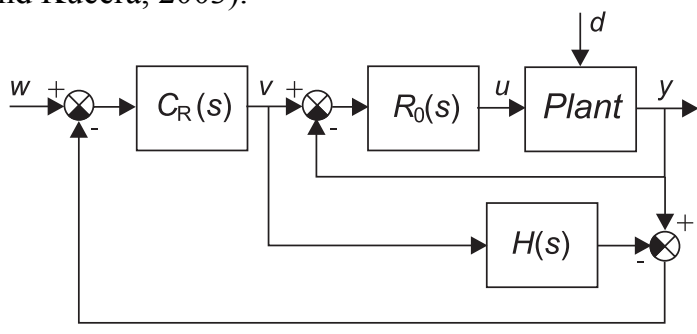

Fig. 2 Affine parametrization of controller $R_{M}(s)$

The key point of affine parameterization (19) consists in the fact that it describes all possible linear time-invariant controllers that render the control system in Fig. 2 internally stable for a stable plant $H(s)$. All that is needed is to ensure that $C_{R}(s)$ is a stable RQ-meromorphic transfer function. A common objective is to shape the output disturbance sensitivity to approach zero for $\omega \rightarrow 0$. On the other hand, the system has to reject measurement noise and modeling errors and that is why its sensitivity functions are required to roll-off at high frequencies. To combine these requirements with the aim of a feasible plant inversion (causal above all), consider the following factorization of the plant $H(s)=H_{0}(s) D(s)$, where $D(s)$ absorbs the noninvertible input delays of the plant and satisfies the condition $D(0)=1$. Then, with respect to (2) and (17) $H_{0}(s)$ has a stable and causal inverse. The parameterizing function $C_{R}(s)$ is taken in the form

$$
C_{R}(s)=F(s) H_{0}^{-1}(s)
$$

where $F(s)$ is selected as a low-pass filter or a stable conditioning factor with a steady state gain of one, $F(0)=1$, that renders $C_{R}(s)$ a stable RQmeromorphic function. Recall that any stable RQmeromorphic function $C_{R}(s)$ is sufficient to establish an internally stable control loop.

Theorem 1. Consider the following factorization of the time delay stable system (18)

$$
H(s)=H_{0}(s) D(s)=\frac{B_{H}(s)}{A_{H}(s)} D(s),
$$

where $A_{H}(s)$ and $B_{H}(s)$ are two coprime stable retarded quasi-polynomials of order $2 n-1$ and $m+n-1, m \leq n$, respectively and $D(s)$ absorbs the non-invertible delays of the $H(s)$ numerator quasipolynomial. If the conditioning factor $F(s)$ is taken to be a proper and stable rational function of the generic form

$$
F(s)=\left(1+f_{1} s+\ldots+f_{n-m} s^{n-m}\right)^{-1}
$$

and if $F(s)$ denominator polynomial is coprime with both $A_{H}(s)$ and $B_{H}(s)$, the parameterizing controller function $C_{R}(s)$ given by (20) is a proper and stable RQ meromorphic function and the controller given by (19)

$$
R_{M}(s)=\frac{F(s) A_{H}(s)}{B_{H}(s)[1-F(s) D(s)]}
$$

is an internally stabilizing controller for the plant $H(s)$.

Proof. Since no multiplicative delay factors are present in $B_{H}(s)$, the function $C_{R}(s)$ that results from (20) as

$$
C_{R}(s)=\frac{A_{H}(s)}{B_{H}(s)\left(1+f_{1} s+\ldots+f_{n-m} s^{n-m}\right)}
$$

is a stable RQ-meromorphic function with the order $2 n-1$ in both numerator and denominator. The resulting feedback controller $R_{M}(s)$ is then given by (19) as follows

$$
R_{M}(s)=\frac{F(s) A_{H}(s)}{B_{H}(s)[1-F(s) D(\mathrm{~s})]}=\frac{Y(s)}{X(s)}
$$

Consider the following expression

$$
A_{H}(s) X(s)+B_{H}(s) D(s) Y(s)=A_{H}(s) B_{H}(s)
$$

and note, with regard to $(25)$, that each term of this equation contains the factor $A_{H}(s) B_{H}(s)$. The inverse of this factor is a stable RQ-meromorphic function. Thus, this factor can be cancelled from equation (26) giving the following result

$$
\begin{aligned}
& \frac{A_{H}(s)}{A_{H}(s) B_{H}(s)} X(s)+\frac{B_{H}(s) D(s)}{A_{H}(s) B_{H}(s)} Y(s)= \\
& =M(s) X(s)+N(s) Y(s)=1
\end{aligned}
$$

Since $N(s) / M(s)=H(s),(27)$ is a Bézout equation related to the control loop and therefore $R_{M}(s)$ is a stabilizing controller for $H(s)$.

Remark. (Disturbance rejection) Since the exact inverse of $H(j \omega)$ cannot be achieved in the parameterizing controller function $C_{R}(j \omega)$, it remains to require an integral character of the controller $R_{M}(s)$, which can be achieved by fulfilling the condition $C_{R}(0)=H^{-1}(0)$. However, this way of formulating the disturbance rejection issue does not cover the requirements concerning the desired frequency band of control performance, which can be satisfied only for suitable parameters of $F(s)$. Nevertheless, the integral character of $R_{M}(s)$ is always achieved by the design above since $C_{R}(0)=A_{H}(0) / B_{H}(0)$. Therefore, with respect to (23) and the property $F(0)=D(0)=1$, it holds that

$$
\lim _{s \rightarrow 0}\left[R_{M}(s)\right]^{-1}=0
$$


Example 2. In Example 1, a stabilizing controller $R_{0}(s)$ has been found for the plant (10). This controller was considered as a pre-compensator only to obtain a stable modified system $H(s)$ to be controlled. From (10) and (11) this system results in the following meromorphic function

$$
H(s)=\frac{(s+\lambda) \exp (-s \tau)}{(a \exp (-s \vartheta))(s+\lambda)+(\delta s+\kappa) \exp (-s \tau)}
$$

where the controller parameters $\lambda, \delta, \kappa$ were found by the pole shifting approach. Before applying the inversion scheme $(20), H(s)$ is factorized as $H(s)=H_{0}(s) \exp (-s \tau)$. The preliminary control function is then

$$
\begin{aligned}
& C_{R}(s)=F(s) H_{0}^{-1}(s)= \\
& \frac{F(s)\left[\left(s^{2}-a \exp (-s \vartheta)\right)(s+\lambda)+(\delta s+\kappa) \exp (-s \tau)\right]}{\delta s+\kappa}
\end{aligned}
$$

To obtain a proper $C_{R}(s)$ it is sufficient to select a second order conditioning factor, say $F(s)=$ $=\left(\alpha^{2}+\Omega^{2}\right) /\left[(s+\alpha)^{2}+\Omega^{2}\right]$, where $\alpha=\xi \Omega$ with a given damping factor $\xi$ (e.g. $\xi=0.5$ ). Then the parameterizing control function is

$$
\begin{aligned}
& C_{R}(s)= \\
& \frac{\left(\alpha^{2}+\Omega^{2}\right)\left[\left(s^{2}-a \exp (-s \vartheta)\right)(s+\lambda)+(\delta s+\kappa) \exp (-s \tau)\right]}{\left[(s+\alpha)^{2}+\Omega^{2}\right](\delta s+\kappa)}
\end{aligned}
$$

The corresponding master controller $R_{M}(s)$ then results from this function as

$$
\begin{gathered}
R_{M}(s)=\frac{C_{R}(s)}{1-C_{R}(s) T(s)}= \\
=\frac{\left(\alpha^{2}+\Omega^{2}\right)\left[\left(s^{2}-a \exp (-s \vartheta)\right)(s+\lambda)+(\delta s+\kappa) \exp (-s \tau)\right]}{\left[(s+\alpha)^{2}+\Omega^{2}-\left(\alpha^{2}+\Omega^{2}\right)\right](\delta s+\kappa)}
\end{gathered}
$$

having at least one pole in $s=0$, since $R_{M}^{-1}(0)=0$. With this anisochronic controller the complementary sensitivity function of the complete double loop control system is as simple as follows

$$
\frac{R_{M}(s) H(s)}{1+R_{M}(s) H(s)}=\frac{\left(\alpha^{2}+\Omega^{2}\right) \exp (-s \tau)}{(s+\alpha)^{2}+\Omega^{2}}
$$

provided the plant model is well fitting the real plant. For a damping high enough, e.g. $\alpha=0.5 \Omega$, the dynamics of control system are tuned only by setting an appropriate product $\tau \Omega$.

\section{CONCLUDING REMARKS}

A pragmatic combination of the pole shifting approach and the internal model inversion principle proves effective to provide for a feasible inverse- based affine controller parameterization. This parameterization results in a straightforward description of stabilizing controllers for both openloop stable and unstable linear time-delay plants. The inverse-based affine parameterization formula (23) facilitates the design of anisochronic controllers for general order quasi-polynomial models without any inherent limitation in this respect. The proposed meromorphic extension of affine controller parameterization inherently involves the additional condition of controller causality, which has been avoided by the separate treatment of the delay factor $D(s)$ in the proposed design procedure. However, the restriction to RQ meromorphic functions reduces the design to retarded time-delay systems only.

Acknowledgement. The research is supported by the Ministry of Education of the Czech Republic under the Project 1 M6840770004.

\section{REFERENCES}

Goodwin, G., C., Graebe, S. F., and Salgado, M. E., 2001 Control System Design. (New Jersey: Prentice Hall)

Hale, J. K., and VerduYn Lunel, S. M., 1993, Introduction to Functional Differential Equations, Vol. 99 of Applied Math. Sciences, (New York: Springer-Verlag, Inc.)

MaleK-ZAVAreI, M., and JAMShIDI, M., 1987, Time Delay Systems: Analysis, Optimization an Application. (Amsterdam: North Holland)

Michiels, W., Engelborghs, K., Vansevenant, P. and Roose, D., 2002, Continuous Pole Placement Method for Delay Equations, Automatica 38, No. 5, pp. 747 - 761

Bonnet C. and Partington, J.R., 2000, Stabilization of Fractional Exponential Systems Including Delays. In: $2^{\text {nd }}$ IFACWorkshop on Linear Time Delay Systems, Ancona.

VYHLÍDAL T. and ZíTEK, P., 2003, Quasi-polynomial Mapping Based Rootfinder for Analysis of Time Delay Systems. In: IFAC Time Delay Systems 03, Rocquencourt

WAN, Q.G., LEE, T.H., and TAN, K.K., 1995, Finite Spectrum Assignment Controllers for Time Delay Systems. (Springer, London)

ZíteK, P., and HlaVA, J., 2001, Anisochronic Internal Model Control of Time Delay Systems. Control Engineering Practice. 9, 2001, No. 5, 501-516

ZíteK, P., VyhlídAL, T., and FiŠER, J., 2001, Anisochronic State Feedback Design Compensating for System Delays. Third IFAC Workshop on Time Delay Systems, Santa Fé

ZÍTEK, P.and VYHLÍDAL, T., 2003, Quasi-Polynomial Based Design of Time Delay Control Systems. Fourth IFAC Workshop on Time Delay Systems, Rocquencourt

ZíteK, P.and KuČera, V., 2003, Algebraic Design of Anisochronic Controllers for Time Delay Systems. Int. J. Control Vol. 76, No. 16, pp. $1654-1665$ 